\title{
Multitype Galton-Watson processes
}

\section{M.P. Quine}

This dissertation is concerned with aspects of the behaviour of multitype Galton-Watson processes. In particular, it contains a complete basic asymptotic theory for processes of this kind, with immigration, which are positively regular. "Heavy traffic" theorems are given for positively regular processes both with and without inmigration. The moment structure is also considered.

Specifically, after an introductory chapter, Chapter II analyzes certain positively regular processes with immigration. Section 1 deals with the subcritical case. A necessary and sufficient condition on the immigration component is given for such a process to have a limiting stationary distribution. Section 2 deals with the supercritical case. It is shown that the process behaves largely as it would if there were no immigration effect (this latter behaviour is described in Kesten and Stigum [2]). In both sections, it is shown how earlier single-type results are improved on by the present work. Some pathologies are discussed in the second section.

Chapter III introduces the notion of a class of "paracritical" processes (without immigration), that is, a class of positively regular processes with $\rho$ near unity, which satisfy certain moment conditions. Theorem 1 gives results concerning the iterates of the generating functions, showing in particular that they can be uniformly approximated by rational linear functions. Theorem 2 gives expressions for the probability that a process is not extinct by the $n$-th generation, and its expected value at this time conditional on non-extinction. The principal result (Theorem 3) indicates that the limit theorem for a critical process, given

Received 13 April 1972. Thesis submitted to the Australian National University, September 1971. Degree approved, March 1972. Supervisors: Dr E. Seneta, Professor P.A.P. Moran. 
by Joffe and Spitzer ([1], Theorem 6), continues to hold in an approximate sense for these paracritical processes. The error estimate is shown to be uniform for all processes within the class which have parameter $\rho$ lying within a given distance of unity.

The first section of Chapter IV completes the triad of theorems for positively reguler processes with immigration, by proving the following result. Consider a critical process with immigration, which has its offspring covariance matrices, and immigration mean vector, elementwise finite. Then, when normed by $n^{-1}$, the process tends in law to a multivariate gamma distribution which is concentrated on a line, whose direction does not depend on the nature of the immigration component. The second section contains the "paracritical" version of this result.

Chapter $V$ examines a different facet of multitype Galton-Watson processes, namely, their moment structure. It is shown that by using Kronecker (direct) products, linear recurrence relations of a simple form can be derived for the first and second (and more in some cases) moments of successive generations. With the additional assumption of positive regularity, these are iterated to give limiting expressions, and rates of convergence, for the first and second moments of both sorts of process, in the three cases $\rho \gtreqless 1$.

\section{References}

[1] A. Joffe and F. Spitzer, "On multitype branching processes with $\rho \leq 1$ ", J. Math. Anal. Appl. 19 (1967), 409-430.

[2] H. Kesten and B.P. Stigum, "A limit theorem for multidimensional Galton-Watson processes", Ann. Math. Statist. 37 (1966), 1211-1223. 